\title{
Large-aperture wide-bandwidth antireflection-coated silicon lenses for millimeter wavelengths
}

\author{
R. Datta, ${ }^{1, *}$ C. D. Munson, ${ }^{1}$ M. D. Niemack, ${ }^{2,3}$ J. J. McMahon, ${ }^{1}$ J. Britton, ${ }^{3}$ E. J. Wollack, ${ }^{4}$ \\ J. Beall, ${ }^{3}$ M. J. Devlin, ${ }^{5}$ J. Fowler, ${ }^{3}$ P. Gallardo, ${ }^{6}$ J. Hubmayr, ${ }^{3}$ K. Irwin, ${ }^{3}$ L. Newburgh, ${ }^{7}$ \\ J. P. Nibarger, ${ }^{8}$ L. Page, ${ }^{7}$ M. A. Quijada, ${ }^{4}$ B. L. Schmitt, ${ }^{5}$ S. T. Staggs, ${ }^{7}$ \\ R. Thornton, ${ }^{9}$ and L. Zhang ${ }^{7}$ \\ 'University of Michigan Physics Department, 450 Church St., Ann Arbor, Michigan 48109, USA \\ ${ }^{2}$ Cornell University Physics Department, 109 Clark Hall, Ithaca, New York 14853, USA \\ ${ }^{3}$ National Institute of Standards and Technology, 325 Broadway, Boulder, Colorado 80305, USA \\ ${ }^{4}$ NASA Goddard Space Flight Center, 8800 Greenbelt Road, Greenbelt, Maryland 20771, USA \\ ${ }^{5}$ University of Pennsylvania Department of Physics and Astronomy, 209 South 33rd St., Philadelphia, Pennsylvania 19104, USA \\ ${ }^{6}$ Departamento de Astronomia y Astrofisica, Facultad de Fisica, Pontificia Universidad Catolica, Casilla 306, Santiago 22, Chile \\ ${ }^{7}$ Princeton University Department of Physics, Jadwin Hall, P.O. Box 708, Princeton, New Jersey 08544, USA \\ ${ }^{8}$ Boulder Micro-Fabrication Facility, National Institute of Standards and Technology, 325 Broadway, \\ MS 817.03, Boulder, Colorado 80305, USA \\ ${ }^{9}$ West Chester University of Pennsylvania Department of Physics, 700 South High St., West Chester, Pennsylvania 19383, USA \\ *Corresponding author: dattar@umich.edu \\ Received 26 July 2013; revised 24 October 2013; accepted 25 October 2013; \\ posted 25 October 2013 (Doc. ID 194154); published 16 December 2013

\begin{abstract}
The increasing scale of cryogenic detector arrays for submillimeter and millimeter wavelength astrophysics has led to the need for large aperture, high index of refraction, low loss, cryogenic refracting optics. Silicon with $n=3.4$, low loss, and high thermal conductivity is a nearly optimal material for these purposes but requires an antireflection (AR) coating with broad bandwidth, low loss, low reflectance, and a matched coefficient of thermal expansion. We present an AR coating for curved silicon optics comprised of subwavelength features cut into the lens surface with a custom three-axis silicon dicing saw. These features constitute a metamaterial that behaves as a simple dielectric coating. We have fabricated silicon lenses as large as $33.4 \mathrm{~cm}$ in diameter with micromachined layers optimized for use between 125 and $165 \mathrm{GHz}$. Our design reduces average reflections to a few tenths of a percent for angles of incidence up to $30^{\circ}$ with low cross polarization. We describe the design, tolerance, manufacture, and measurements of these coatings and present measurements of the optical properties of silicon at millimeter wavelengths at cryogenic and room temperatures. This coating and lens fabrication approach is applicable from centimeter to submillimeter wavelengths and can be used to fabricate coatings with greater than octave bandwidth. () 2013 Optical Society of America

OCIS codes: $\quad$ (000.2190) Experimental physics; (220.0220) Optical design and fabrication; (220.3630) Lenses; (310.1210) Antireflection coatings; (160.1245) Artificially engineered materials; (310.6628) Subwavelength structures, nanostructures.

http://dx.doi.org/10.1364/AO.52.008747
\end{abstract}

$1559-128 \mathrm{X} / 13 / 368747-12 \$ 15.00 / 0$

(C) 2013 Optical Society of America

\section{Introduction}

The development of large format superconducting detector arrays for millimeter and submillimeter 
astrophysics (e.g., [1-3]) has driven the need for highthroughput optical designs that maintain diffraction limited performance across the arrays (see [4] for a review). Silicon is an excellent material for these applications due to its high index of refraction $(n=3.4)$, low loss-tangent ( $\tan \delta<7 \times 10^{-5}$ at $4 \mathrm{~K}$ ), and relatively high thermal conductivity $\left(k \sim 200 \mathrm{Wm}^{-1} \mathrm{~K}^{-1}\right.$ at $4 \mathrm{~K}$ [5], which is only a factor of 50 lower than high quality oxygen free high conductivity (OFHC) copper [6] and orders of magnitude higher than plastic). The essential development required to realize optical designs using silicon optics at millimeter wavelengths is an appropriate antireflection (AR) coating to mitigate the $30 \%(-5 \mathrm{~dB})$ reflective loss from each optical surface.

AR coatings consist of one or more dielectric layers placed on the surface of refractive optical elements. The indices of refraction and thicknesses of the AR layers are chosen such that the reflections from the vacuum-AR interface and from the $A R$-substrate interface interfere and cancel. If the optical path length through a single-layer AR coating is one quarter wavelength, the refractive index of the coating is $\sqrt{n}$ (the geometric mean of the substrate index and free space), and the coating is free from dielectric losses, the cancellation is perfect at one particular frequency. Applied to silicon, such quarter-wave coatings reduce reflections below 1\% over 1.25:1 bandwidth at normal incidence.

Wider bandwidths and larger ranges of angles of incidence can be accommodated by adding additional layers to the AR coating to form a multilayer coating. These coatings require layers with a number of different refractive indices. Cryogenic applications require that the coefficients of thermal expansion of the AR layers are sufficiently matched to that of the lens substrate to prevent damage upon cooling. The dielectric loss and birefringence of the AR coating material must also be carefully controlled. Locating materials with these properties poses a significant challenge to implementing cryogenic wide band AR coatings for silicon. One avenue to solve this problem is to engineer materials with the required dielectric constant by cutting subwavelength features into the lens surface $[7,8]$. Engineered optical materials with properties determined by their detailed geometric shape, size, and orientation are referred to as "artificial dielectric metamaterials."

These metamaterial coatings consist of layers of subwavelength holes [9], posts, or grooves [10] cut into the substrate to be coated. This approach allows the index of the AR layer to be precisely tuned by adjusting the geometry of the machined features, naturally solves the thermal expansion problem, and has loss lower than that of the substrate. At cryogenic temperatures silicon has a dielectric loss tangent 100 times lower than typical plastics like Cirlex, which have previously found use as singlelayer AR coatings [11]. Rosen et al. [12] developed an epoxy-based multilayer broadband $\bar{A} R$ coating for millimeter-wave cryogenic optics. The advantages of silicon metamaterial AR layers include precisely controlled indices of refraction, inherently matched thermal expansion, and significantly reduced dielectric losses.

This approach has been successfully applied to plastic millimeter-wave optics through direct machining and silicon optics for visible wavelengths through patterning of geometric structures which behave as an AR coating similar to that found on a moth's eye [13-15]. At millimeter wavelengths Zhang et al. [16] fabricated an alternative class of artificial dielectric AR coatings for silicon composed of metal resonant structures supported by a plastic substrate that had higher losses than the AR layers described here. Schuster et al. [17] fabricated single-layer silicon micromachined artificial dielectrics, and Han et al. [18] have used silicon immersion grating technology to fabricate planar artificial dielectrics for terahertz radiation. Our work is the first demonstration of broadbandwidth metamaterial AR layer on silicon optics with finite curvature for millimeter wavelengths.

The AR coated lenses presented here have been developed for the ACTPol [19] project, a polarization sensitive receiver for the Atacama Cosmology Telescope (ACT, [20]). In Section 2 we present the design requirements for the ACTPol optics as they apply to this work. The design of the AR layers is described in Section 3. In Section 4 we describe the ACTPol lens design. Cryogenic measurements of the dielectric properties of silicon samples taken from $45 \mathrm{~cm}$ diameter ingots at millimeter wavelengths are presented in Section 5 . In Section 6 we describe the fabrication process of the AR coatings on the lenses and present reflection measurements of a finished lens. We conclude with a brief discussion of the range of applicability of this coating technique.

\section{Requirements}

The performance requirements for the AR coated silicon lenses described in this work are dictated by the scientific goals of the ACTPol project [19]. This instrument is designed to detect the faint polarized signals from the cosmic microwave background (CMB) using three detector arrays fed by independent reimaging optics. Two of the arrays are horn coupled polarimeters for the $150 \mathrm{GHz}$ band (passband from 125 to $165 \mathrm{GHz}$ ), while the third array will be a multichroic array including both 150 and $90 \mathrm{GHz}$ bands ("90 $\mathrm{GHz}$ " passband from 80 to $110 \mathrm{GHz}$ ) [21]. Extensive design studies demonstrated high index of refraction $(n \gtrsim 3)$ lenses were needed to achieve high optical quality across the required field of view.

In this paper we focus on the AR coatings for the $150 \mathrm{GHz}$ band lenses. These are conceptually similar to those which will be used for the broader-band multichroic lenses. The bandwidth requirement for the $150 \mathrm{GHz}$ band coatings is to have $>99 \%$ transmission between 125 and $165 \mathrm{GHz}$. The left panel of Fig. 1 shows the reimaging optics for a $150 \mathrm{GHz}$ array. The optics include three plano-convex silicon lenses with 

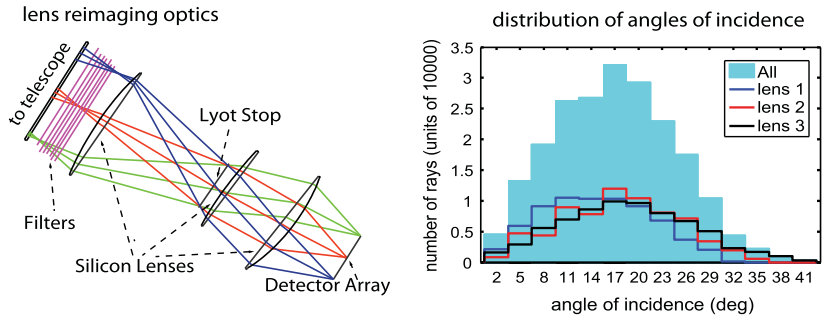

Fig. 1. Left: Ray diagram of one set of ACTPol reimaging optics, which includes three silicon lenses feeding a detector array. Right: Histogram of the angles of incidence of rays at the surfaces of these three silicon lenses.

diameters up to $33.4 \mathrm{~cm}$. The figure shows that rays passing through the optical system refract over a wide range of angles of incidence. This is quantified in the right-hand panel of Fig. 1 , which shows that the distribution of angles of incidence is centered near $17^{\circ}$ and that more than $96 \%$ of the rays have angles of incidence $<30^{\circ}$. This sets the requirement that the coatings must be optimized to minimize reflections for angles of incidence between $0^{\circ}$ and $30^{\circ}$.

Since ACTPol is a polarization sensitive experiment, low cross polarization is another requirement. Studies of polarization systematics (e.g., [22]) suggest that the CMB temperature to polarization leakage must be controlled to better than $1 \%$, which corresponds to a requirement that differences in the transmission for the two polarizations be $0.5 \%$.

To reduce the thermal emission, all optics are cooled to $4 \mathrm{~K}$ or below. Thus the AR coating must be able to withstand cryogenic cycling.

As described in Section 5, high resistivity silicon, which is available in boules up to $45 \mathrm{~cm}$ in diameter, offers a cryogenic loss tangent $\tan \delta<7 \times 10^{-5}$, an index of refraction of $n=3.4$, and a relatively high thermal conductivity, making it an ideal material for this optical design. Our approach of directly machining metamaterial AR layers into the lens surfaces guarantees that the layers have low dielectric losses and coefficients of thermal expansion that are inherently matched to that of the silicon lenses.

\section{AR Coating Design}

The AR coating is comprised of two metamaterial layers. Each layer consists of an array of square pillars on a square grid cut using silicon dicing saw blades by making two sets of parallel trenches rotated $90^{\circ}$ relative to each other. It has been shown that any electromagnetic structure with an axis of $n$-fold $(n>2)$ rotational symmetry must have polarization independent reflectance and zero cross polarization when light is incident along this axis [23]. Hence the rotational symmetry of these layers leads to zero cross polarization at normal incidence and low birefringence at oblique incident angles. Figure 2 shows a cross section and isometric view of this geometry which is parameterized by the depths $d_{1}$, $d_{2}$ and kerf widths $k_{1}, k_{2}$ of the inner and outer layer, respectively, and a pitch $p$. The outer layer is cut by using a blade of width $k_{2}$ and the inner layer is
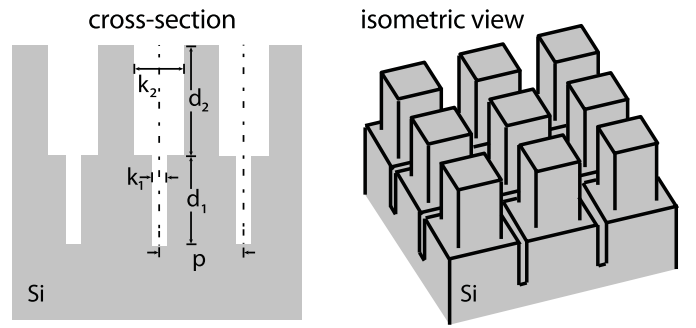

Fig. 2. Geometry of the two-layer metamaterial AR coating. The left side shows a cross section through the center of the pillars indicating the design parameters discussed in the text. The right side shows an isometric view of the structure resulting from making cuts along two perpendicular directions.

cut by making a second set of cuts to greater depth with a thinner blade of width $k_{1}$. We introduce the volume fill factor $v_{f}=(1-k / p)^{2}$ to facilitate comparison to published analytic calculations based on the second order effective medium theory developed by Rytov [24] for the effective dielectric constant in the quasi-static limit $(p \ll \lambda)[15,25-27]$.

The metamaterial layers can be treated as a volume distribution of small electromagnetic scatterers characterized by electric and magnetic polarizability densities [7]. When the pitch is small compared to the shortest wavelength of interest, the fields in the layer are homogenous [8] and one can define an effective dielectric constant which can be used to parameterize the propagation properties of the media. This effective dielectric function for the layer, $\epsilon_{r}^{\text {eff }}$, is a function of the density of scatterers in the layer $[28,29]$, parametrized by the volume fill factor. As the feature size of a composite medium approaches a significant fraction of the radiation wavelength, the effective dielectric function becomes frequency dependent [30]. As the wavelength is further reduced the artificial dielectric structure no longer appears homogenous and these simple quasi-static considerations must be augmented to adequately model its behavior. In going beyond the quasi-static limit resonant effects, diffraction and scattering can occur in the artificial dielectric structure [9,31-33]. The AR coatings described here, which operate between the quasi-static and diffractive regimes, rely on numerical simulations to estimate the relation between the volume fill factor and the effective refractive index of the metamaterial layer.

The values of $d_{1}, d_{2}, k_{1}, k_{2}$, and $p$ were chosen using a three-step design process. A preliminary design was carried out using a classical analytic model consisting of sheets of dielectric material each with a constant thickness and index of refraction [34]. These were varied to minimize the reflectance across the band yielding targets for the electrical thickness and index of refraction of our two-layer coating.

The second step was to translate the index and thickness from the simple model into the pitch, kerfs, and depths for our geometry. This step required knowledge of the relation between the effective index of refraction of an array of square pillars and the 


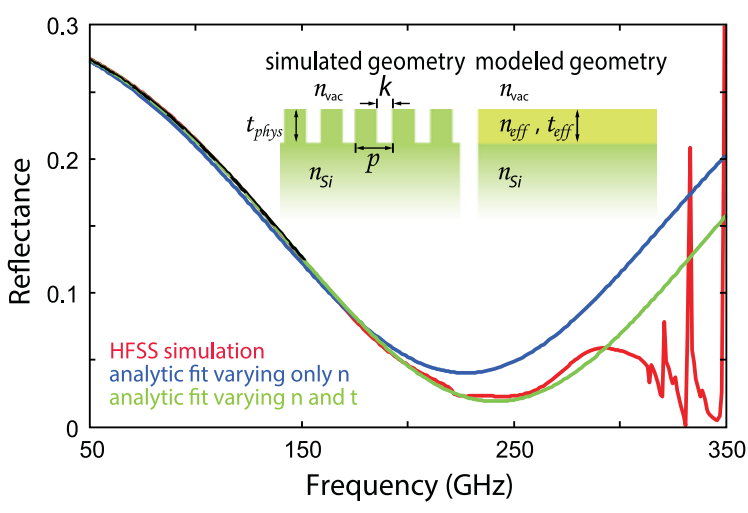

Fig. 3. Comparison of the reflectance calculated from an HFSS numerical simulation of a pillar geometry to fits based on a model of a simple dielectric layer. The geometry is specified in the inset in the top. In the simulated geometry, $p=400 \mu \mathrm{m}, t_{\mathrm{phys}}=220 \mu \mathrm{m}$, $k=40 \mu \mathrm{m}$, and $n_{\mathrm{Si}}=3.38$. A comparison between fits where only the index $n$ is free and where both the index $n$ and thickness $t$ are varied is shown. The HFSS simulation and the best fit curve differ by $5 \%$ at $\approx 220 \mathrm{GHz}(1.36 \mathrm{~mm})$ for the analytic fit varying $n$ and $t$ and at $\approx 180 \mathrm{GHz}(1.67 \mathrm{~mm})$ for the analytic fit varying only $n$. This corresponds to the minimum wavelength, $\lambda_{5 \%}$, (and a corresponding maximum frequency $f_{5 \%}$ ) for the specified pitch, $p$, below (above) which the layer no longer behaves as a simple dielectric layer.

pitch and kerf of that layer. These relations were determined by fitting analytic models to high frequency structure simulator (HFSS, [35]) simulations of single layers with a wide range of volume fill factors for a few different choices of pitch. The HFSS simulations were carried out using Floquet ports and master-slave boundary conditions to model a box containing a single post of the metamaterial as an infinite periodic two-dimensional array of features [35].

Figure 3 shows a representative simulation of a single-layer pillar geometry with insets showing the simulated geometry and the simple dielectric layer modeled in the fit. The fit constrained the effective index $n_{\text {eff }}$, the effective electrical thickness $t_{\text {eff }}$, and the maximum frequency to which our metamaterial layer behaves as a simple dielectric. For the purposes of our fit we define this maximum frequency as the point where the simulated reflectance and the analytic fit disagree at more than 5\% absolute reflectance. We refer to this frequency as $f_{5 \%}$ and the corresponding wavelength $\lambda_{5 \%}$. This indicates the transition between specular and diffractive behavior of the layer.

A second fit which fixed the electrical thickness to the physical thickness of the pillars is also shown in Fig. 3. This fit implies a lower $f_{5 \%}$. The discrepancy between the best fit thickness $t_{\text {eff }}$ and the physical thickness $t_{\text {phys }}$ of the metamaterial layer arises since the geometric structure of the pillars results in fringing of fields at the junction between layers, causing a perturbative shift between the position of the physical interface and the effective location of the junction's electrical reference plane. An analogous effect is encountered in the design of metallic waveguide structures [32], which can be analytically treated.

Figure 4 shows the results from fitting a number of HFSS simulations with different pitch and fill factors to the analytic model. Plots include the breakdown wavelength $\lambda_{5 \%}$, the effective index $n_{\text {eff }}$, and the electrical thickness of a layer with index $\sqrt{n_{\mathrm{Si}}}$ expressed as a ratio of the thickness of a quarter-wave homogenous dielectric layer and a quarter-wave metamaterial layer.

The recovered $\lambda_{5 \%}$ is in good agreement with the analytic condition $[\underline{9}, \underline{33}]$ to prevent diffraction from a grating array,

$$
p<\frac{\lambda}{\left(n_{s}+n_{i} \sin \theta_{i}\right)}
$$

where $p$ is the pitch, $\lambda$ is the wavelength, $n_{s}$ is the index of the substrate, $n_{i}$ is the index of the incident medium, and $\theta_{i}$ is the angle of incidence. This analytic expression shows that the pitch must be chosen based on the indices of the adjacent media and must be smaller than implied by the $\lambda_{5 \%}$ calculated at normal incidence to minimize diffraction at oblique angles of incidence. Consideration of manufacturing cost and mechanical robustness of the two-layer AR design favors the largest possible pitch, as this choice minimizes the number of cuts required to cover a given area and
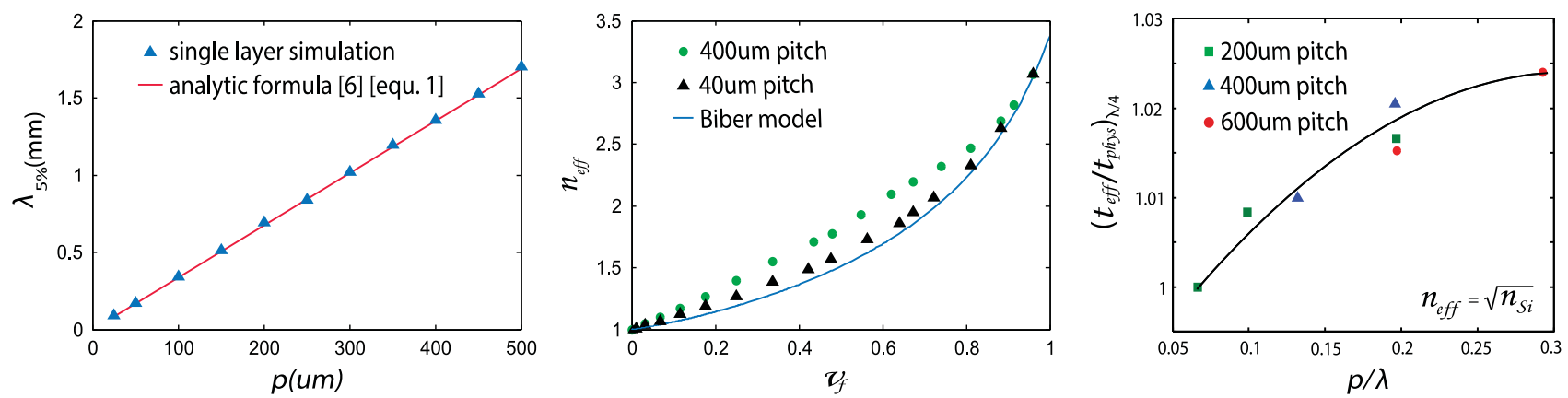

Fig. 4. Summary of results from HFSS simulations of single layers of pillars with varying geometry. Left: Minimum wavelength $\lambda_{5} \%$ for which the single pillar layer is fit by an analytic model to $5 \%$ accuracy as a function of pitch $p$ at normal incidence. Center: effective index of refraction $n_{\text {eff }}$ as a function of the volume fill factor $v_{f}$ obtained by fitting the simulated reflectances up to the minimum wavelength $\lambda_{5 \%}=$ $1360 \mu \mathrm{m}$ for a pitch $p=400 \mu \mathrm{m}, 40 \mu \mathrm{m}$. The line labeled "Biber model" is a prediction from the analytic Biber model [25], which corresponds to the quasi-static circuit approximation for the media. Right: Thickness $t_{\text {eff }}$ for a slab dielectric quarter-wave layer divided by the thickness $t_{\mathrm{phys}}$ of pillars forming an effective quarter-wave coating as a function of $p / \lambda$. The index of the simulated dielectric layer was held fixed at $\sqrt{n_{\mathrm{Si}}}$ for these simulations. A quadratic fit is plotted to guide the eye. 
makes the pillars larger and therefore stronger. A $435 \mu \mathrm{m}$ pitch leads to acceptable performance up to $\sim 175 \mathrm{GHz}(\sim 1.7 \mathrm{~mm})$, which is the upper edge of our band.

Comparing this relation between $v_{f}$ and $n_{\text {eff }}$ to analytic models $[15,25]$ for the case where the electric field of the incident wave is perpendicular to the grooves, we found discrepancies that are reduced as the pitch decreases and we approach the quasistatic limit $p / \lambda \ll 0.1$. Given that our design does not operate in this limit and simple analytic models have insufficient accuracy for our purposes, we resort to numerical simulation to optimize the geometry of our AR layers.

The variation in the electrical thickness compared to the physical thickness (Fig. 4, right) is shown for the case of a quarter-wave AR layer with index $\sqrt{n_{\mathrm{Si}}}$. Additional simulations show that the electrical thickness depends on the index of refraction of the material on either side of the metamaterial dielectric layer. We account for this small effect in the final numerical optimization of the multilayer design.

With these relations (Fig. 4) in hand we convert our two-layer coating into the desired parameters for the pillar geometry. In the final step we performed a numerical optimization of the AR layers at $15^{\circ}$ angle of incidence using HFSS, with the constraint that the cut geometry must match the kerf geometry of commercially available dicing saws (see Section 6). This step ensures manufacturability, accounts for any discrepancies between the electrical and physical thickness implied by our numerical simulations, and improves the performance at larger angles of incidence. Table 1 gives the parameters for the resultant design, and Fig. 5 shows the simulated performance for this AR design as a function of frequency for several $\left(0^{\circ}, 15^{\circ}\right.$, and $\left.30^{\circ}\right)$ angles of incidence. Even at $30^{\circ}$ incidence the band averaged reflections are at $-26 \mathrm{~dB}$ and differences between the two linear polarization states (TE and TM; see [34] for definition) are below $0.5 \%$ for all frequencies. At $15^{\circ}$ incidence, average reflections are below $-31 \mathrm{~dB}$. The tolerance of the design to manufacturing errors are described in detail in Appendix A.

\section{ACTPol Lenses}

The ACTPol lenses are cylindrically symmetric plano-convex designs in which the convex surface

Table 1. Parameters for the ACTPol Metamaterial Coating

\begin{tabular}{lccc}
\hline Parameter & Symbol & $\begin{array}{c}\text { Dimension } \\
(\mu \mathrm{m})\end{array}$ & $\begin{array}{c}\text { Dimension } \\
\text { (units of } p \text { ) }\end{array}$ \\
\hline Pitch & $p$ & 435 & 1.0 \\
Kerf $^{a}$ & $k_{1}$ & 41 & 0.094 \\
& $k_{2}$ & 190 & 0.437 \\
Depth & $d_{1}$ & 217 & 0.499 \\
& $d_{2}$ & 349 & 0.802 \\
Volume fill & $v_{f 1}$ & 0.820 & \\
$\quad$ factor & & & \\
& $v_{f 2}$ & 0.317 & \\
\hline
\end{tabular}

${ }^{a}$ Nominal average values.
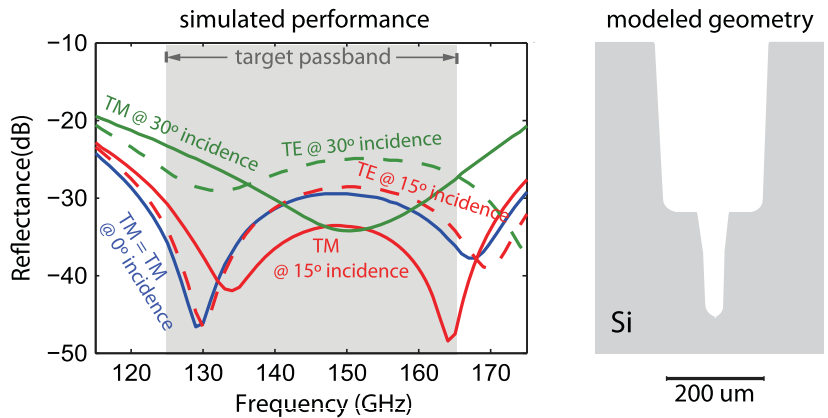

Fig. 5. Left: Simulation of the performance of the ACTPol coating at a range of angles of incidence. Right: Unit cell geometry (based on measured cut profiles) modeled using HFSS.

is aspheric-a conic section with four perturbing terms proportional to the fourth, sixth, eighth, and tenth orders of the distance from the axis. The design optimization procedure for the reimaging optics is similar to that described in [20], the primary difference being that the ACTPol optics are required to be image-space telecentric to optimize the coupling to the planar feedhorn arrays. This was accomplished by constraining the chief rays at each field point to be near normal incidence at the focal plane and by allowing the tilts of the lenses and the focal plane to vary during the optimization. The resulting design achieves Strehl ratios greater than 0.93 across the $150 \mathrm{GHz}$ focal planes without accounting for the Gaussian illumination of the feedhorns, which effectively improves the image quality. All three of the ACTPol optics tubes use the same three silicon lens designs with the positions and tilts adjusted to optimize the coupling to the ACT Gregorian telescope [20].

The AR coating approach we have developed constrains the perimeter of the lens designs to enable accurate clamping during both machining of the AR coatings and cryogenic cycling of the lenses. Figure 6 shows a cross section of the perimeter of an ACTPol lens design. Each lens includes a handling ring along its perimeter that is not AR coated and is used for mounting the lens during machining as well as clamping to cool the lens in the cryogenic receiver. The outer corners of the perimeter are chamfered to minimize chipping. There are steps rising from the perimeter to the lens surface to provide clearance between the perimeter clamping region and the AR layers. The lens blanks were machined by $\mathrm{Nu}$-Tek

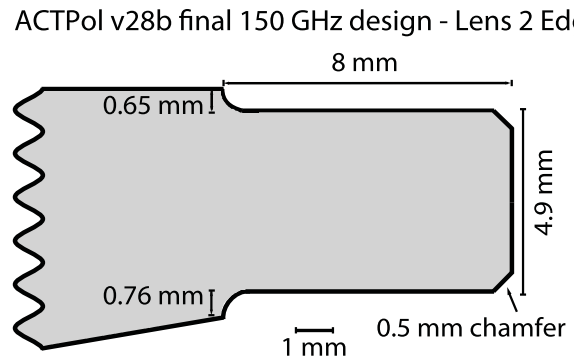

Fig. 6. Cross section of the perimeter of the ACTPol lens 2, which is designed to enable machining of the AR layers and clamping the lens without damaging the AR coatings. 
Precision Optical Corporation and achieved $5 \mu \mathrm{m}$ tolerances.

\section{Properties of Silicon}

Silicon manufacturing can produce different grades of material, including ultrahigh purity silicon produced by the float zone process [36] that has a negligible density of impurities and silicon produced by the Czochralski process [36] that has a higher level of impurities. As charge carriers and associated states introduced by impurities are the cause of dielectric loss, use of the highest available purity (as inferred from the room temperature resistivity) silicon minimizes the dielectric losses. Measurements of the refractive index, dielectric permittivity, and loss tangent of various high purity and high resistivity silicon samples over a range of frequencies and temperature have been reported [37-39]. For example, ultrahigh purity silicon has been measured to have a loss tangent of $\sim 1 \times 10^{-5}$ at room temperature which would correspond to $<1 \%$ loss in the ACTPol optical system. Unfortunately, surface tension limits the zone-melt purification technique used to produce ultrahigh purity silicon to diameters below about $200 \mathrm{~mm}$. Therefore, the substrates available for large diameter lenses considered in this work must be fabricated from Czochralski silicon. For our low temperature application we expect the bulk conductivity of silicon to freeze out, dramatically reducing the dielectric losses; however, other loss mechanisms can persist. We validate this general picture of the dielectric loss in silicon by optically characterizing the influence of the bulk resistivity as a function of frequency and sample temperature.

Samples produced by the Czochralski process and readily available in $450 \mathrm{~mm}$ diameter stock with bulk resistivities specified to be in the range of 1 to $>500 \Omega$-cm were characterized both at room temperature and $4 \mathrm{~K}$ using a Bruker 125 high-resolution Fourier transform spectrometer (FTS) with a Oxford Cryostat CF continuous liquid helium flow sample chamber. The cryostat is equipped with pair of $75 \mu \mathrm{m}$ thick polypropylene windows that enable spectral measurement while allowing the sample to be held at a regulated temperature. The samples were cut to have a typical thickness of $180 \mu \mathrm{m}$, double side polished, and placed in a $25 \mathrm{~mm}$ diameter optical test fixture at the focus of an $f / 6$ beam. The reflections from the two surfaces of the sample form a FabryPerot resonator for which the modeling is relatively simple, permitting measurement of dielectric properties. The silicon samples are boron doped ( $p$-type) to adjust the resistivity. The $>500 \Omega$-cm resistivity silicon used in the ACTPol lenses has the minimum dopant level.

Each sample's transmission was measured between $240 \mathrm{GHz}\left(8 \mathrm{~cm}^{-1}\right)$ and $18 \mathrm{THz}\left(600 \mathrm{~cm}^{-1}\right)$ using different combinations of sources, beam splitters, and detectors for three frequency bands of $240-450 \mathrm{GHz}$ $\left(8-15 \mathrm{~cm}^{-1}\right), \quad 450-2850 \mathrm{GHz} \quad\left(15-95 \mathrm{~cm}^{-1}\right)$, and $2.85-18 \mathrm{THz}\left(95-600 \mathrm{~cm}^{-1}\right)$.
The spectral resolution employed, $7.5 \mathrm{GHz}$ $\left(0.25 \mathrm{~cm}^{-1}\right)$, fully resolves the sample's spectral features. Sliding stages permit the sample or a reference clear aperture to be moved into the FTS beam while in the cryostat for in situ calibration.

The transmission spectrum was modeled as a series of homogeneous plane parallel dielectric layers [40]. The dielectric function for the silicon was approximated by a classical Drude dispersion model [41]:

$$
\varepsilon_{r}^{*}(\omega)=\varepsilon_{\infty}^{*}-\frac{\omega_{p}^{2}}{\omega \cdot(\omega+i \Gamma)},
$$

where $\varepsilon_{r}^{*}=\varepsilon_{r}^{\prime}+i \varepsilon_{r}^{\prime \prime}$ is a complex function of frequency $\omega$, the damping rate $\Gamma$, and the contribution to the relative permittivity $\varepsilon_{\infty}^{*}$ of higher energy transitions. The plasma frequency and the damping rates are related by $\omega_{p}^{2}=\Gamma / \varepsilon_{o} \rho$, where $\varepsilon_{o}$ is the permittivity of free space and $\rho$ is the sample's bulk resistivity [42]. In this approximation, the material's free carriers are treated as classical point charges undergoing random collisions, and the resulting damping is assumed to be independent of the carrier energy. We find this representation suitable to represent the sample's properties over the spectral and temperature ranges of interest.

The sample thickness is known to $\pm 0.5 \mu \mathrm{m}$ at room temperature and corrected for thermal contraction [43]. Since the fringe rate is proportional to the product of the refractive index and sample thickness, uncertainties in the sample thickness directly limit the precision of the determination of $\Re\left(\varepsilon_{\infty}^{*}\right)$. Measurements of an optically polished crystalline quartz sample with accurately known thickness were used to measure a $1 \%$ amplitude uncertainty across the entire FTS band. This calibration uncertainty leads to a corresponding reduction in the measurement's sensitivity to $\mathfrak{s}\left(\varepsilon_{\infty}^{*}\right)$. These uncertainties were accounted for in fitting the FTS data. These fits produced root-mean-square deviations in the range of $\sim 0.005$ to 0.016 between the model and the observation spectra. Representative data for the $\rho>500$ and $1<\rho<5 \Omega$-cm samples are shown in Fig. 7 for sample physical temperatures of $T_{\text {phys }}=297$ and $4.5 \mathrm{~K}$.

The results of these fits are shown in Table 2. As anticipated the finite plasma frequency and damping rate contribute significantly to the room temperature losses; however, the $\rho>500 \Omega$-cm sample could be approximated by a dielectric constant over the spectral range of interest (e.g., $\Gamma$ and $\omega_{p}$ are consistent with zero). The influence of free carrier collisions at room temperature on the optical response becomes more pronounced in the $1<\rho<5 \Omega$-cm sample, manifesting as a reduction in transmission at low frequencies. However, as the $1<\rho<5 \Omega$-cm sample is cooled, bulk conduction is suppressed, and this effect shifts to lower frequencies than those of interest for millimeter-wave applications. The observed dielectric parameters are a weak function of temperature below $\sim 30 \mathrm{~K}$, as anticipated given the exponential thermal dependence of the bulk resistivity [44]. 


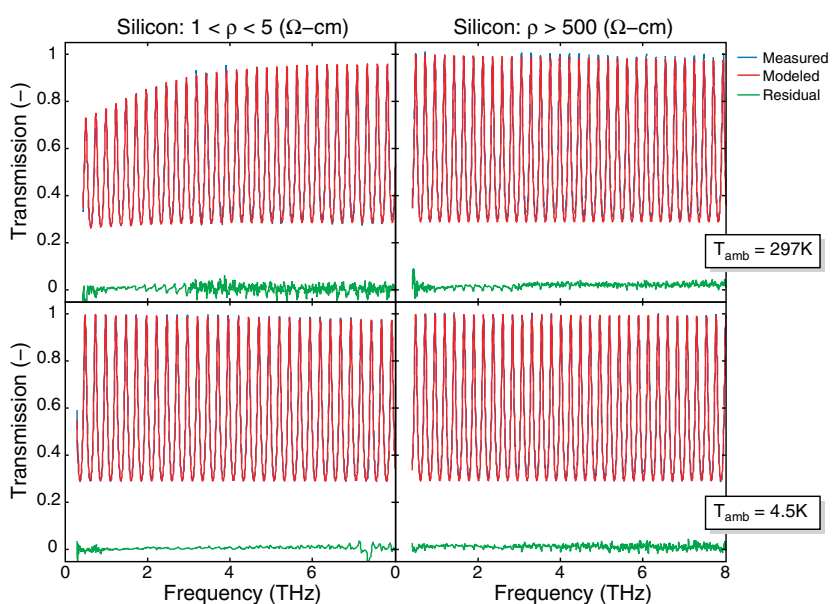

Fig. 7. Measured silicon transmission at room temperature (upper panels) and $4.5 \mathrm{~K}$ (lower panels). The left and right columns in the panel are for samples specified as $1<\rho<5 \Omega$-cm and $\rho>500 \Omega$-cm, respectively; the sample thickness is $\sim 180 \mu \mathrm{m}$. The figure contains the measured FTS data (red), model (dashed blue), and residual (green).

Both samples show $\sim 1 \%$ shifts in the magnitude of the index of refraction $\left(n \approx \sqrt{\varepsilon_{\infty}^{\prime}}\right)$ upon cooling. This shift is accounted for in our optical design. At low temperature both the high and low resistivity sample give an upper limit for the loss tangent of $\tan \delta \sim \varepsilon_{\infty}^{\prime \prime} / \varepsilon_{\infty}^{\prime}<7 \times 10^{-5}$. For the ACTPol optics at $150 \mathrm{GHz}$, this corresponds to an absorptive loss of $<5 \%$ for our optical system comprising three lenses. The ACTPol lenses were fabricated from $\rho>$ $500 \Omega$-cm silicon as it may reduce loss and facilitates room temperature testing. Our models and observations suggest that the carriers will be frozen out at $4 \mathrm{~K}$ for both high and low resistivity silicon, but given the small cost differential high resistivity silicon is the natural choice. We note that the conductivity of silicon at room temperature, and therefore the loss, is a strong function of UV irradiance. We have not investigated this dependence cryogenically.

\section{Fabrication}

The AR coatings were fabricated using silicon dicing saw blades, which are available in widths ranging from 10 to hundreds of micrometers. Mounted on a commercial silicon dicing saw machine, these blades

Table 2. Silicon Drude Model Fit Parameters

\begin{tabular}{|c|c|c|c|c|c|}
\hline $\begin{array}{l}\rho\left(T_{\text {phys }}\right) \\
{[\Omega-\mathrm{cm}]}\end{array}$ & $\begin{array}{c}T_{\text {phys }} \\
{[\mathrm{K}]}\end{array}$ & $\begin{array}{l}\varepsilon_{\infty}^{\prime} \\
{[-]}\end{array}$ & $\begin{array}{l}\varepsilon_{\infty}^{\prime \prime} \\
{[-]}\end{array}$ & $\begin{array}{c}\Gamma / 2 \pi \\
{[\mathrm{THz}]}\end{array}$ & $\begin{array}{l}\omega_{p} / 2 \pi \\
{[\mathrm{THz}]}\end{array}$ \\
\hline \multirow[t]{2}{*}{$>500$} & 297 & $11.7(1)$ & 0.0015 & - & - \\
\hline & 4.5 & $11.5(5)$ & $<0.0008$ & - & - \\
\hline \multirow[t]{7}{*}{1 to 5} & 297 & $11.6(55)$ & 0.0046 & 0.571 & 1.60 \\
\hline & 200 & $11.5(52)$ & 0.0049 & 0.572 & 0.80 \\
\hline & 100 & $11.4(78)$ & 0.0028 & 0.459 & 0.26 \\
\hline & 70 & $11.4(66)$ & 0.0011 & 0.162 & 0.20 \\
\hline & 30 & $11.4(64)$ & $<0.0008$ & - & - \\
\hline & 10 & $11.4(62)$ & $<0.0008$ & - & - \\
\hline & 4.5 & $11.4(62)$ & $<0.0008$ & - & 一 \\
\hline
\end{tabular}

repeatedly cut with micrometer-level precision and cutting speeds up to several centimeters per second. Unfortunately, commercial silicon dicing machines are not designed for curved surfaces and cannot accommodate the large diameter lenses required for this project. Therefore we constructed a custom three-axis silicon dicing system to fabricate these structures.

The fabrication system is shown in Fig. $\underline{8}$. It consists of a micrometer accurate three axis stage on which we mount an air bearing dicing spindle and a micrometer accurate depth gauge. The spindle and retractable depth gauge are attached to the vertical stage ( $z$ axis), which rides on the horizontal ( $y$ axis) stage. A lens is mounted on an aluminum mounting plate on a horizontal stage ( $x$ axis) below the spindle. This plate permits the lens to be accurately rotated by $0^{\circ}, 90^{\circ}, 180^{\circ}$, and $270^{\circ}$ and carries a reference wafer that is used in setting up the blades prior to cuts. A side-looking microscope is mounted parallel to the $y$ axis to characterize test cuts on the reference wafer. Flood cooling water is sprayed on the dicing blade while cuts are made to carry away debris. A temperature controlled water bath is used to regulate the temperature of the spindle and maintain the flood coolant and air surrounding the dicing system within $1^{\circ} \mathrm{C}$.

Our system does not show any appreciable change in cut shape or surface damage when cutting at the maximum travel speed of $50 \mathrm{~mm} / \mathrm{s}$. We conservatively operate at $25 \mathrm{~mm} / \mathrm{s}$, for which it takes a total of $12 \mathrm{~h}$ of machining per lens side. Factoring in setup time it takes six 8-h days to fabricate a single lens.

Figure 9 shows photographs of one of the ACTPol $\mathrm{AR}$ coated silicon lenses. The fabricated features are sufficiently robust to permit handling the lenses by touching the AR coatings. The manufacture resulted in fewer than 10 out of 500,000 posts with damage visible by eye. The shape of the cut profiles was evaluated by making cuts on a reference silicon wafer before cutting (precut) and after cutting (postcut) the lens and measuring them using the side-looking microscope. In addition, reference cuts were examined at frequent intervals during the process of cutting

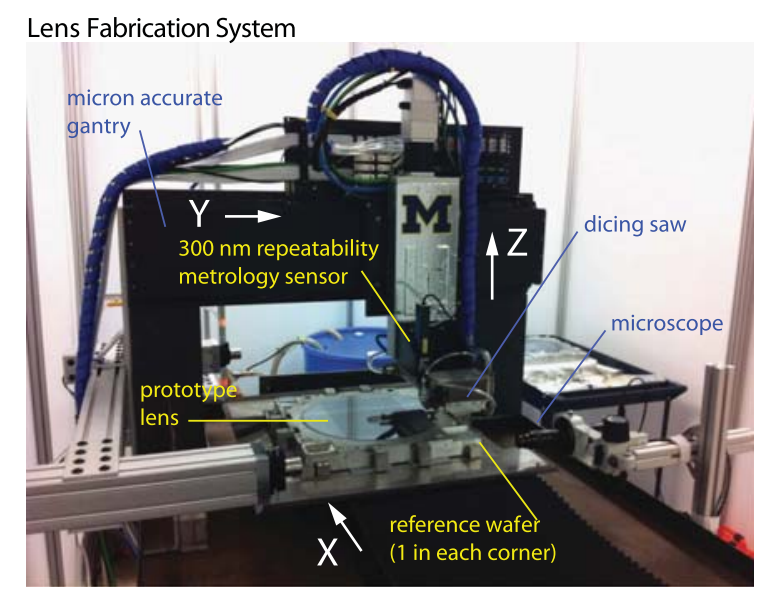

Fig. 8. The custom, micrometer accurate, three axis silicon dicing system used to fabricate AR coatings on the lenses. The labels identify the key components and axes described in Section $\underline{6}$. 

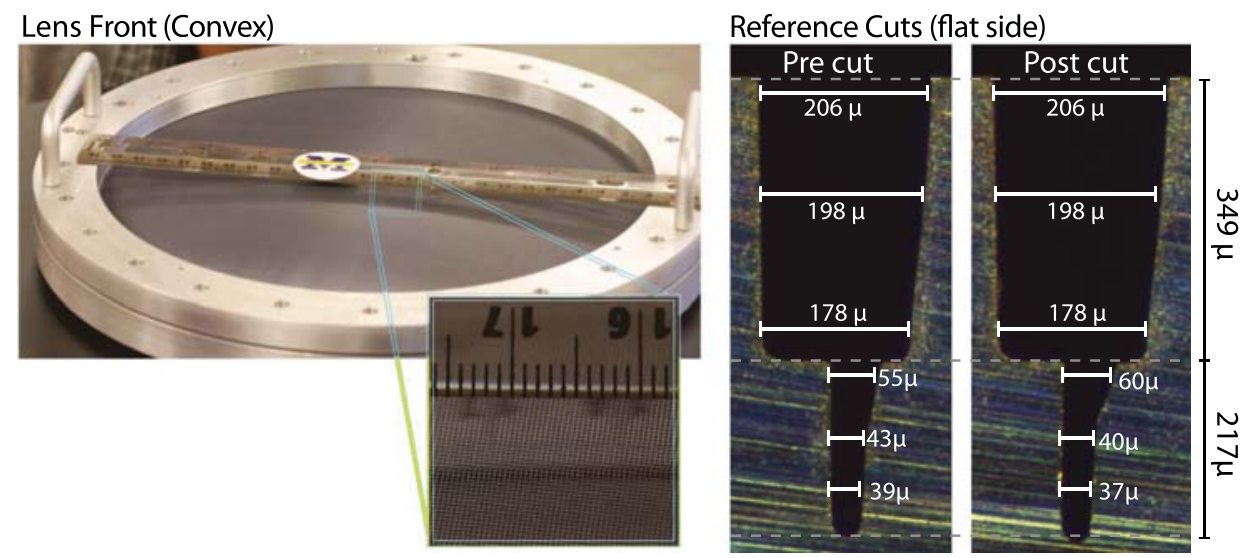

Fig. 9. Left: Photograph of the curved front surface of an AR coated ACTPol silicon lens (lens 2) with zoomed view of a small patch. Right: Photographs taken with side-looking metrology microscope of a reference wafer taken prior to and after cutting the coatings on a lens.

the lens, and the whole range of profiles were found to be within acceptable tolerance limits. Comparison of the precut and postcut measurements (see Fig. 9) show that the wide blades cut repeatably with negligible evolution to cut profile while the narrow blades show some evolution in width in the upper third of the cut. Simulations show that this evolution leads to a few tenths of a percent increase in the reflectance. The accuracy of the depths of the cuts is limited by a $3 \mu \mathrm{m}$ uncertainty in the zero point for the blade depth and $\mathrm{a} \pm 7 \mu \mathrm{m}$ surface error in the lens. This surface error is due to imperfections in the lens mounting plate that stress and warp the lens.

The reflectance of the completed ACTPol lenses were measured using a scalar reflectometer. This reflectometer consisted of a tunable narrowband continuous wave source which illuminated the flat side of lens through a $20^{\circ}$ full width half power horn, from a distance of $5 \mathrm{~cm}$, and tilted at an angle of $15^{\circ}$ relative to the lens surface normal. A receiver consisting of an identical horn coupled to a detector diode was placed at the mirror image of the source horn relative to the plane defined by the point of maximum illumination of the lens and perpendicular to a line joining the source and receiver. This system was calibrated by (1) placing an aluminum reflector at the same position as the lens to normalize the peak reflection to unity, and (2) by removing the lens and calibration reflector to measure the stray reflections which were found to be negligible.

The results of this reflectance measurement are shown in Fig. 10 for both the TE and TM polarizations. Precise modeling of this measurement configuration requires accounting for the interference between reflections from the flat and curved lens surfaces, which is beyond the scope of this work. However, the results are expected to be intermediate between the reflectance from a single AR coated surface (single sided) and the easily simulated result for two flat coated surfaces separated by the central thickness of the lens (two sided). These two cases are presented in Fig. 10 for both linear polarization states. These simulations incorporate the measured

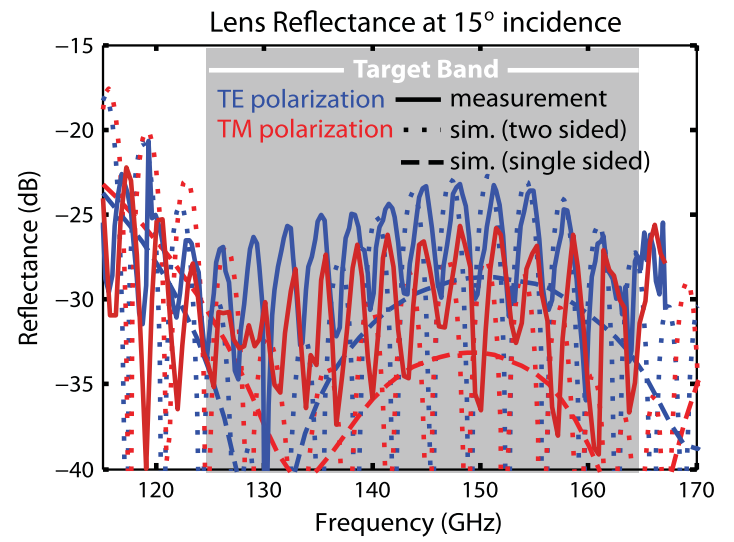

Fig. 10. Comparison between simulations and measurements of the fabricated lens. The two-sided simulations model the AR coatings on both sides of a flat silicon sample of thickness equal to that of the lens at its center. The single-sided simulations model the AR coatings on only one side of a flat silicon sample.

cut profiles shown in Fig. 9 . We empirically found that moving the lens so that it is well centered on the beam increases the interference effects (TM case) while moving the lens off center reduces these effects (TE case). Based on the reasonable agreement between these simplistic simulations and the measurement we are confident we are reducing reflections to a few tenths of a percent over the range of angles of incidence required for ACTPol.

\section{Conclusion}

We have described a new approach for AR coating silicon lenses over broad bandwidths and a range of angles of incidence. Simulations, backed up by measurements of an AR coated lens, show that the fabricated coatings presented here reduce reflections below a few tenths of a percent between 125 and $165 \mathrm{GHz}$ for angles of incidence between 0 and $30^{\circ}$ for cryogenic applications. We have developed a micrometer accurate three axis silicon dicing saw facility and are using it to manufacture AR coated lenses for ACTPol. We have shown that a range of $p$-type silicon doping levels can achieve low loss at 
cryogenic temperatures using silicon samples from boules as large as $45 \mathrm{~cm}$ diameter. This approach for implementing wide-bandwidth AR coated silicon lenses is applicable for millimeter and submillimeter wavelength ranges and can be expanded to wider bandwidth by adding additional layers.

\section{Appendix A: Tolerances}

The manufacturing tolerances for the eight types of machining errors shown in Fig. 11 were evaluated based on a fiducial design. These include (1) errors in the pitch, $\delta p ;(2)$ errors in the centering of the deeper groove relative to the shallow groove, $\delta c$; (3) errors in the kerf widths, $\delta k_{1}, \delta k_{2}$; (4) slopes in the walls of the grooves parameterized by $\delta_{S 1}$ and $\delta_{S 2}$, which represent the distance the upper and lower grooves tilt inward at the groove bottom if the mean width is fixed; (5) errors in the depth of either groove, $\delta d_{1}, \delta d_{2}$; (6) differences in the depth of grooves in the two orthogonal directions assuming the correct mean depths are parameterized $\delta d_{\mathrm{LR}_{1}}$ for the upper and $\delta d_{\mathrm{LR}_{2}}$ for the lower groove; (7) fabricating layers on a surface tilted at an angle $\phi$ (see Fig. 11) and where the depth of each groove is measured at the center of the groove (e.g., the effect of applying these coatings at the edge of a curved lens); and (8) rows of broken posts. The tolerance to variation in the refractive index of the silicon substrate, $\delta n_{\mathrm{Si}}$, was also evaluated. The tolerances for these parameters were quantified based on a fiducial model similar to what was fabricated but with straight-walled pillars using HFSS simulations in which the parameters in each family were varied separately. For the majority of these effects the results were distilled to a band averaged reflection at $15^{\circ}$ angles of incidence. The sensitivity to each parameter was quantified as the displacement needed to bring about a $3 \mathrm{~dB}$

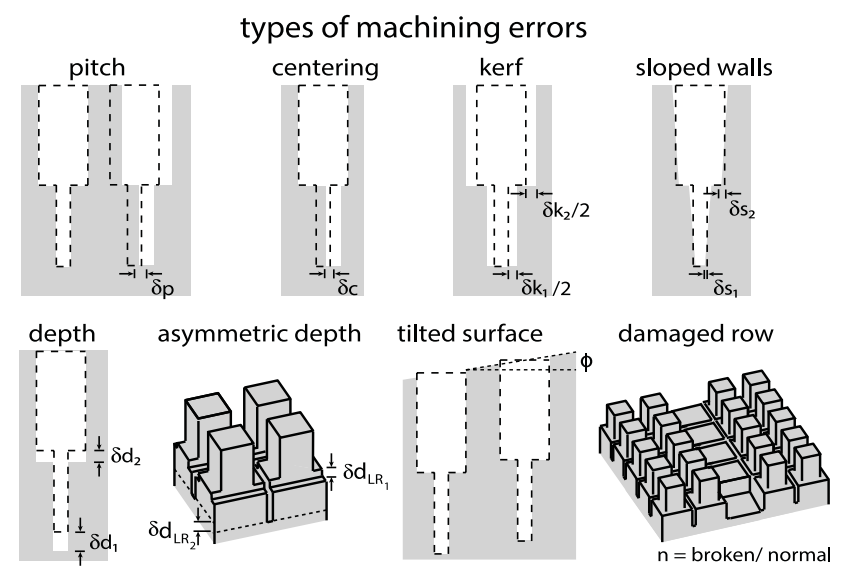

Fig. 11. This figure shows the families of machining errors considered in the tolerance analysis and described in the text. The dashed lines show the target shape for the grooves and that for the pillars while the white space in the gray represents the material as actually cut. Therefore difference between the dashed region and the white region represent machining errors. For the asymmetric depth and damaged row errors the sketches show the geometry of the errors. increase in reflectance. For the case of tilted surface and for broken posts the results of a small number of simulations were evaluated and compared to the fiducial performance. In the remainder of this section we discuss these results.

\section{A. Pitch}

Variations in the pitch change the effective index of both layers. Figure 12 shows the band averaged reflectance as function of variation in the pitch. A conservative tolerance of $\delta p<3 \mu \mathrm{m}(\delta p / p<0.0075)$ limits the change in reflectance to be less than $3 \mathrm{~dB}$.

\section{B. Centering}

The performance was insensitive to errors in the centering of the deeper grooves within the shallower grooves. This is consistent with both layers of pillars behaving as layers with a constant effective index of refraction. Aperiodic misalignments of the deeper grooves within the shallow grooves are equivalent to variation in the pitch.

\section{Kerf}

The upper left panel of Fig. 13 presents the impact of errors in the kerf. Controlling $\delta k_{1}$ and $\delta k_{2}$ to $3 \mu \mathrm{m}$ ensures a less than $3 \mathrm{~dB}$ degradation in performance. Varying both parameters in the same direction (e.g., $\delta k_{1}=\delta k_{2}$ ) has little impact on the performance. We have chosen the fiducial design to be near the high end of the most favorable region to make the manufacture relatively immune to blade wear, which could have (but did not) narrow the kerf width as machining proceeds.

\section{Sloped Walls}

The upper-right panel of Fig. 13 presents the impact of slopes in the walls left behind by the dicing saw. Conservative estimates for the $3 \mathrm{~dB}$ tolerance for these two parameters are $6 \mu \mathrm{m}$ for $\delta s_{1}$ and $3 \mu \mathrm{m}$ for $\delta s_{2}$. Sloping both layers by a similar amount results in negligible degradation.

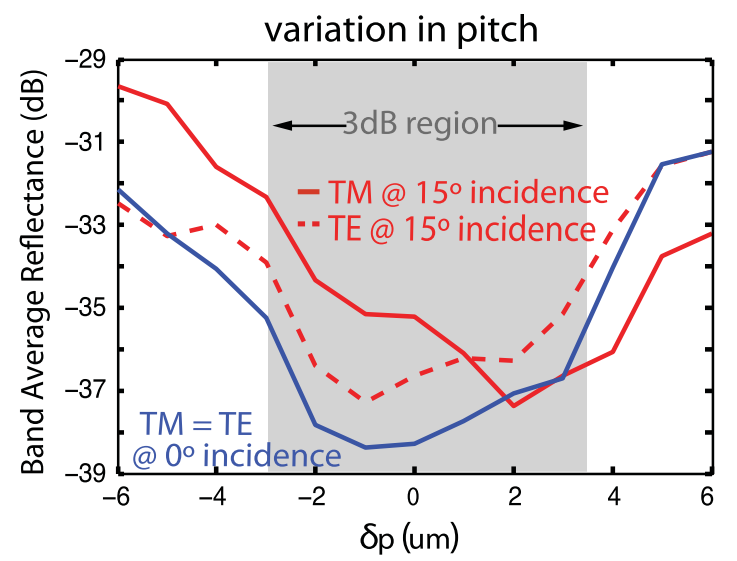

Fig. 12. Band averaged reflectance at a $15^{\circ}$ angle of incidence as a function of changes in the pitch. The gray region shows range for which the performance is within $3 \mathrm{~dB}$ of the fiducial design. 

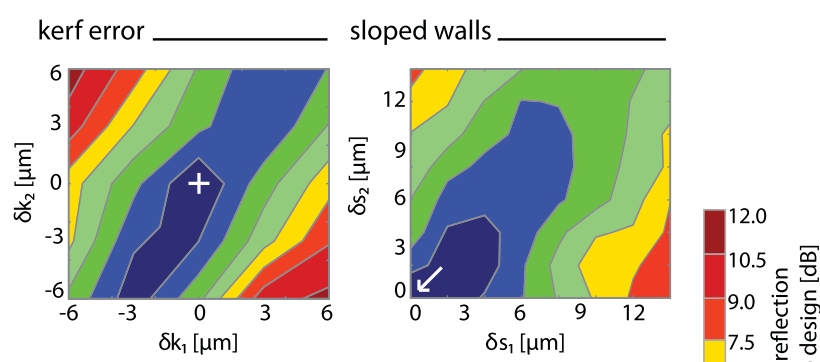

depth error

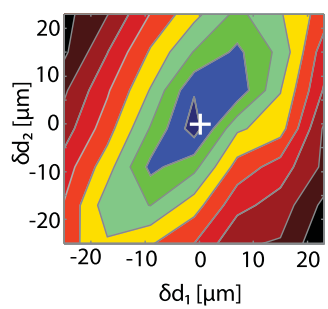

asymmetric depth

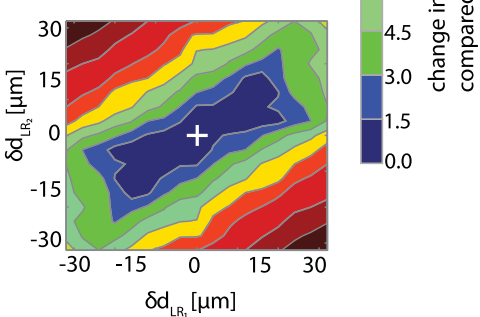

Fig. 13. This figure shows the impact of errors in the kerf, slopes in the sidewalls, overall depth errors, and asymmetric differences in depth between the two orthogonal cuts. The color scale presents the degradation in reflection at $15^{\circ}$ incidence compared to the simulations in Fig. 5. The fiducial design is at the coordinate $(0,0)$ in all the plots as highlighted by a "+" or arrow. The outer edge of the light blue contour (see label) represents $3 \mathrm{~dB}$ degradation. The horizontal (vertical) axis represents errors in the inner (outer) layer for the labeled parameters.

\section{E. Depth}

The lower left panel of Fig. 13 presents the impact of depth errors. The $3 \mathrm{~dB}$ tolerances for these parameters are $7.5 \mu \mathrm{m}$ for $\delta d_{1}$ and $10 \mu \mathrm{m}$ for $\delta d_{2}$. Expressed in terms of optical path length the sensitivities are identical.

\section{F. Asymmetric Depth}

The lower right panel of Fig. 13 presents the impact of asymmetric depth. The $3 \overline{\mathrm{dB}}$ tolerances for these parameters are $15 \mu \mathrm{m}$ for $\delta d_{\mathrm{LR}_{1}}$ and $12 \mu \mathrm{m}$ for $\delta d_{\mathrm{LR}_{2}}$ for asymmetry in directions common to both grooves. This error also affects the $90^{\circ}$ rotational symmetry of the geometry resulting in birefringence, but the cross polarization can be no larger than the absolute reflection.

\section{G. Application to Tilted Surfaces}

The left panel of Fig. 14 compares the performance of these layers cut on a surface tilted by $10^{\circ}$ compared with the performance when cut on a flat surface. Fortuitously, this effect improves performance at large angles of incidence and has little effect at small angles. Applying this coating by making grooves parallel to the lens symmetry axis of a curved lens will produce acceptable performance.

\section{H. Groups of Broken Posts}

Assessing the impact of the broken posts is not straightforward because the region and extent of illumination varies between the three lenses. The degradation due to broken posts would thus be a function of the effective aperture area of the lenses
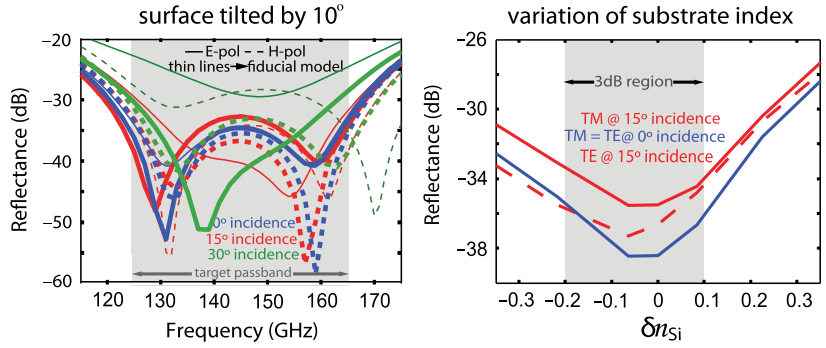

Fig. 14. Left: Simulated impact of having the surface of the lens tilted by $10^{\circ}$. Right: Simulated impact of variation in the refractive index of the silicon substrate.

and the location of the defects in addition to the total area affected. Accurately quantifying this is beyond the scope of this work. Qualitatively, the degradation would be negligible if the fraction of total area affected is small and, more importantly, the length scale of the individual affected areas is smaller than the wavelength of incident light. Simulating the impact of broken posts is computationally difficult. Therefore we resorted to a simplified model consisting of a unit cell containing seven posts in a row with one broken off completely. This is equivalent to having one-seventh of the posts destroyed. This pessimistic case produces band averaged reflections below $2 \%$, which is acceptable, though it does produce nontrivial cross polarization. We used simulation to estimate that keeping the number of broken posts below $1 / 700$ would result in a negligible degradation in the overall performance of the AR coating.

\section{Index}

Figure 14, right, shows the band averaged reflectance as a function of variation in the index. A conservative tolerance for the index is $\delta n_{\mathrm{Si}}<0.1\left(\delta n_{\mathrm{Si}} / n_{\mathrm{Si}}<0.03\right)$. This leads to increases in the reflectance of less than $3 \mathrm{~dB}$ over the fiducial design. This tolerance is weaker than that imposed by the optical design.

The $3 \mathrm{~dB}$ sensitivities derived in this section are summarized in Table 3 . The band averaged reflectivity of the fiducial design is $-31 \mathrm{~dB}$ at $15^{\circ}$ incidence,

Table 3. Summary of the $3 \mathrm{~dB}$ Sensitivities for All Parameters Described in the Text

\begin{tabular}{lccc}
\hline Machining Error & Parameter & $\begin{array}{c}\text { Sensitivity } \\
(\mu \mathrm{m})\end{array}$ & $\begin{array}{c}\text { Tolerance } \\
(\text { units of } p)\end{array}$ \\
\hline Pitch $(p)$ & $\delta p$ & 3 & $7.5 \times 10^{-3}$ \\
Centering & $\delta c$ & See text & \\
Kerf & $\delta k_{1}$ & 3 & $7.5 \times 10^{-3}$ \\
& $\delta k_{2}$ & 3 & $7.5 \times 10^{-3}$ \\
Sloped walls & $\delta s_{1}$ & & $1.5 \times 10^{-2}$ \\
& $\delta s_{2}$ & 3 & $7.5 \times 10^{-3}$ \\
Depth & $\delta d_{1}$ & 7.5 & $1.9 \times 10^{-2}$ \\
Asymmetric & $\delta d_{2}$ & 10 & $2.5 \times 10^{-2}$ \\
$\quad$ depth & $\delta d_{\mathrm{LR}_{1}}$ & 15 & $3.8 \times 10^{-2}$ \\
Tilted surface & $\delta d_{\mathrm{LR}_{2}}$ & 12 & $3 \times 10^{-2}$ \\
Damaged row & $\phi$ & See text & \\
Index & $r$ & $\sim 1 / 700$ & $\mathrm{NA}$ \\
\hline
\end{tabular}


which is significantly better than the requirements for the system.

This work was supported by the U.S. National Science Foundation through awards AST-0965625 and PHY-1214379 and by NASA through the NASA Space Technology Research Fellowship training grant NNX12AM32H. The authors would like to thank Ki Won Yoon and Molly Dee for useful discussions.

\section{References}

1. D. Bintley, M. MacIntosh, W. Holland, J. Dempsey, P. Friberg, H. Thomas, P. Ade, R. Sudiwala, K. Irwin, G. Hilton, M. Niemack, M. Amiri, E. Chapin, and M. Halpern, "Commissioning SCUBA-2 at JCMT and optimising the performance of the superconducting TES arrays," J. Low Temp. Phys. 167, 152-160 (2012).

2. M. D. Niemack, Y. Zhao, E. Wollack, R. Thornton, E. R. Switzer, D. S. Swetz, S. T. Staggs, L. Page, O. Stryzak, H. Moseley, T. A. Marriage, M. Limon, J. M. Lau, J. Klein, M. Kaul, N. Jarosik, K. D. Irwin, A. D. Hincks, G. C. Hilton, M. Halpern, J. W. Fowler, R. P. Fisher, R. Dunner, W. B. Doriese, S. R. Dicker, M. J. Devlin, J. Chervenak, B. Burger, E. S. Battistelli, J. Appel, M. Amiri, C. Allen, and A. M. Aboobaker, "A kilopixel array of TES bolometers for ACT: development, testing, and first light," J. Low Temp. Phys. 151, 690-696 (2008).

3. S. Padin, Z. Staniszewski, R. Keisler, M. Joy, A. A. Stark, P. A. R. Ade, K. A. Aird, B. A. Benson, L. E. Bleem, J. E. Carlstrom, C. L. Chang, T. M. Crawford, A. T. Crites, M. A. Dobbs, N. W. Halverson, S. Heimsath, R. E. Hills, W. L. Holzapfel, C. Lawrie, A. T. Lee, E. M. Leitch, J. Leong, W. Lu, M. Lueker, J. J. McMahon, S. S. Meyer, J. J. Mohr, T. E. Montroy, T. Plagge, C. Pryke, J. E. Ruhl, K. K. Schaffer, E. Shirokoff, H. G. Spieler, and J. D. Vieira, "South Pole Telescope optics," Appl. Opt. 47, 4418-4428 (2008).

4. S. Hanany, M. D. Niemack, and L. Page, "CMB telescopes and optical systems," arXiv:1206.2402 (2012).

5. J. C. Thompson and B. A. Younglove, "Thermal conductivity of silicon at low temperatures," J. Phys. Chem. Solids 20, 146-149 (1961).

6. S. W. Va Sciver, Helium Cryogenics, International Cryogenics Monograph Series (Springer Science+Business Media, 2012), Chap. 2.

7. R. E. Collin, Field Theory of Guided Waves (McGraw-Hill, 1990), pp. 749-786.

8. D. R. Smith and J. B. Pendry, "Homogenization of metamaterials by field averaging (invited paper)," J. Opt. Soc. Am. B 23, 391-403 (2006).

9. P.-S. Kildal, K. Jakobsen, and K. Sudhakar Rao, "Meniscuslens-corrected corrugated horn: a compact feed for a Cassegrain antenna," IEE Proc. H Microwaves Opt. Antennas 131, 390-394 (1984).

10. S. B. Cohn, Lens Type Radiators: Antenna Engineering Handbook (McGraw-Hill, 1961).

11. J. Lau, J. Fowler, T. Marriage, L. Page, J. Leong, E. Wishnow, R. Henry, E. Wollack, M. Halpern, D. Marsden, and G. Marsden, "Millimeter-wave antireflection coating for cryogenic silicon lenses," Appl. Opt. 45, 3746-3751 (2006).

12. D. Rosen, A. Suzuki, B. Keating, W. Krantz, A. T. Lee, E. Quealy, P. L. Richards, P. Siritanasak, and W. Walker, "Epoxy-based broadband anti-reflection coating for millimeter-wave optics," arXiv:1307.7827 (2013).

13. P. B. Clapham and M. C. Hutley, "Reduction of lens reflection by 'moth eye' principle," Nature 244, 281-282 (1973).

14. B. S. Thornton, "Limit of the moth's eye principle and other impedance-matching corrugations for solar-absorber design," J. Opt. Soc. Am. 65, 267-270 (1975).

15. M. E. Motamedi, W. H. Southwell, and W. J. Gunning, "Antireflection surfaces in silicon using binary optics technology," Appl. Opt. 31, 4371-4376 (1992).

16. J. Zhang, P. A. R. Ade, P. Mauskopf, L. Moncelsi, G. Savini, and N. Whitehouse, "New artificial dielectric metamaterial and its application as a terahertz antireflection coating," Appl. Opt. 48, 6635-6642 (2009).

17. K. F. Schuster, N. Krebs, Y. Guillaud, F. Mattiocco, M. Kornberg, and A. Poglitsch, "Micro-machined quasi-optical elements for $\mathrm{THz}$ applications," Sixteenth International Symposium on Space Terahertz Technology, Chalmers University of Technology, Gšteborg, Sweden, May 2-4, 2005, pp. 524-528.

18. P. Han, Y. W. Chen, and X.-C. Zhang, "Application of silicon micropyramid structures for antireflection of terahertz waves," IEEE J. Sel. Top. Quantum Electron. 16, 338-343 (2010).

19. M. D. Niemack, P. A. R. Ade, J. Aguirre, F. Barrientos, J. A. Beall, J. R. Bond, J. Britton, H. M. Cho, S. Das, M. J. Devlin, S. Dicker, J. Dunkley, R. Dunner, J. W. Fowler, A. Hajian, M. Halpern, M. Hasselfield, G. C. Hilton, M. Hilton, J. Hubmayr, J. P. Hughes, L. Infante, K. D. Irwin, N. Jarosik, J. Klein, A Kosowsky, T. A. Marriage, J. McMahon, F. Menanteau, K. Moodley, J. P. Nibarger, M. R. Nolta, L. A. Page, B. Partridge, E. D. Reese, J. Sievers, D. N. Spergel, S. T. Staggs, R. Thornton, C. Tucker, E. Wollack, and K. W. Yoon, "ACTPol: a polarization sensitive receiver for the Atacama Cosmology Telescope," Proc. SPIE 7741, 77411S (2010).

20. J. W. Fowler, M. D. Niemack, S. R. Dicker, A. M. Aboobaker, P. A. R. Ade, E. S. Battistelli, M. J. Devlin, R. P. Fisher, M. Halpern, P. C. Hargrave, A. D. Hincks, M. Kaul, J. Klein, J. M. Lau, M. Limon, T. A. Marriage, P. D. Mauskopf, L. Page, S. T. Staggs, D. S. Swetz, E. R. Switzer, R. J. Thornton, and C. E. Tucker, "Optical design of the Atacama Cosmology Telescope and the Millimeter Bolometric Array Camera," Appl. Opt. 46, 3444-3454 (2007).

21. J. J. McMahon, J. Beall, D. Becker, H. M. Cho, R. Datta, A. Fox, N. Halverson, J. Hubmayr, K. Irwin, J. Nibarger, M. Niemack, and H. Smith, "Multi-chroic feed-horn coupled TES polarimeters," J. Low Temp. Phys. 167, 879-884 (2012).

22. M. Shimon, B. Keating, N. Ponthieu, and E. Hivon, "CMB polarization systematics due to beam asymmetry: impact on inflationary science," Phys. Rev. D77, 083003 (2008).

23. A. MacKay, "Proof of polarization independence and nonexistence of crosspolar terms for targets presenting $n$-fold $(n>2)$ rotational symmetry with special reference to frequencyselective surfaces," Electron. Lett. 25, 1624-1625 (1989).

24. S. Rytov, "The electromagnetic properties of finely layered medium," Sov. Phys. JETP 2, 466-475 (1956).

25. S. Biber, J. Richter, S. Martius, and L. P. Schmidt, "Design of artificial dielectrics for anti-reflection-coatings," 33rd European Microwave Conference, Munich, 2003.

26. D. L. Brundrett, E. N. Glytsis, and T. K. Gaylord, "Homogeneous layer models for high-spatial-frequency dielectric surface-relief gratings: conical diffraction and antireflection designs," Appl. Opt. 33, 2695-2706 (1994).

27. T. K. Gaylord, W. E. Baird, and M. G. Moharam, "Zeroreflectivity high spatial-frequency rectangular-groove dielectric surface-relief gratings," Appl. Opt. 25, 4562-4567 (1986).

28. D. E. Aspnes, "Local-field effects and effective-medium theory: a microscopic perspective," Am. J. Phys. 50, 704-709 (1982).

29. D. E. Aspnes, "Bounds on allowed values of the effective dielectric function of two-component composites at finite frequencies," Phys. Rev. B 25, 1358-1361 (1982).

30. W. G. Egan and D. E. Aspnes, "Finite-wavelength effects in composite media," Phys. Rev. B 26, 5313-5321 (1982).

31. A. Wagner-Gentner, U. U. Graf, D. Rabanus, and K. Jacobs, "Low loss THz window," Infrared Phys. Technol. 48, 249-253 (2006).

32. G. Matthaei, L. Young, and E. M. T. Jones, Microwave Filters, Impedance-Matching Networks and Coupling Structures (McGraw-Hill, 1964), pp. 300-304.

33. D. H. Raguin and G. M. Morris, "Analysis of antireflectionstructured surfaces with continuous one-dimensional surfaces profiles," Appl. Opt. 32, 2582-2598 (1993).

34. J. D. Jackson, Classical Electrodynamics (Wiley, 1998).

35. "Ansoft High Frequency Structure Simulator (HFSS) software package," http://www.ansys.com/Products/Simulation+ Technology/Electromagnetics/High-Performance+Electronic+ Design/ANSYS+HFSS. 
36. T. Duffar, Crystal Growth Processes Based on Capillarity: Czochralski, Floating Zone, Shaping and Crucible Techniques (Wiley, 2010).

37. J. Krupka, J. Breeze, A. Centeno, N. Alford, T. Claussen, and L. Jensen, "Measurements of permittivity, dielectric loss tangent, and resistivity of float-zone silicon at microwave frequencies," IEEE Trans. Microwave Theor. Tech. 54, 3995-4000 (2006).

38. M. N. Afsar and H. Chi, "Millimeter wave complex refractive index, complex dielectric constant, and loss tangent of extra high purity and compensated silicon," Int. J. Infrared Millim. Waves 15, 1181-1188 (1994)

39. V. V. Parshin, R. Heidinger, B. A. Andreev, A. V. Gusev, and V. B. Shmagin, "Silicon as an advanced window material for high power gyrotrons," Int. J. Infrared Millim. Waves 16, 863-877 (1995).

40. P. Yeh, Optical Waves in Layered Media (Wiley, 1988).

41. F. Gervais, "High-temperature infrared reflectivity spectroscopy by scanning interferometry," in Electromagnetic Waves in Matter, K. J. Button, ed., Part I, Vol. 8 of Infrared, and Millimeter Waves (Academic, 1983), pp. 284-287.

42. M. Van Exter and D. Grischkowsky, "Optical and electronic properties of doped silicon from 0.1 to $2 \mathrm{THz}$," Appl. Phys. Lett. 56, 1694-1696 (1990).

43. Y. Okada and Y. Tokumaru, "Precise determination of lattice parameter and thermal expansion coefficient of silicon between 300 and 1500 K," J. Appl. Phys. 56, 314-320 (1984).

44. B. I. Shklovskii and A. L. Efros, Electronic Properties of Doped Semiconductors (Springer, 1984), Chap. 4. 